\title{
Second post-Newtonian approximation of light propagation in Einstein-Aether theory $\dagger$
}

\author{
Y. Xie ${ }^{1}$ and T. Y. Huang ${ }^{2}$ \\ Astronomy Department, Nanjing University, \\ Nanjing 210093, 22 Hankou Road, Nanjing, China \\ ${ }^{1}$ email: yixie@nju.edu.cn ${ }^{2}$ email: tyhuang@nju.edu.cn
}

\begin{abstract}
Future deep space laser ranging missions together with astrometry missions will be able to test relativistic gravity to an unprecedented level of accuracy and will require second post-Newtonian approximation of relevant theories of gravity. Einstein-aether theory is adopted as the theory of gravity and the second post-Newtonian approximation of light propagation is studied.
\end{abstract}

Keywords. astrometry, reference systems

\section{Introduction}

Future deep space laser ranging missions and astrometry missions will be able to test relativistic gravity to an unprecedented level of accuracy. More precisely, these missions will enable us to test relativistic gravity to $10^{-6}-10^{-9}$ and will require second postNewtonian $(2 \mathrm{PN})$ approximation of relevant theories of gravity.

Recently, Einstein-Aether theory (ae-theory), which is a kind of vector-tensor theory with a unit time-like vector, is widely discussed in its violation of Lorentz symmetry and broadly applied to astrophysics (Jacobson \& Mattingly 2001, 2004, Eling \& Jacobson 2006, Garfinkle et al. 2007, etc). Furthermore, a vector-tensor theory can show a more comprehensive picture of $2 \mathrm{PN}$ approximation than the scalar-tensor theories (Xie et al. 2007 ) and provides new information for the future parametrized $2 \mathrm{PN}$ metric.

In this paper, we adopt ae-theory as the gravitational theory and derive its $2 \mathrm{PN}$ approximation and equations of light propagation in a $N$ point masses model as a simplified treatment for a real solar system in barycentric reference system.

\section{2PN Approximation of Ae-theory}

In a general tensor-vector theory of gravity, the Lagrangian scalar density involves the metric $g_{\mu \nu}$ and a 4 -vector field $K_{\mu}$. The action defining the theory reads

$$
\begin{aligned}
S=\frac{c^{3}}{16 \pi G} \int[ & R-c_{1} K^{\mu ; \nu} K_{\mu ; \nu}-c_{2} K_{; \mu}^{\mu} K_{; \nu}^{\nu}-c_{3}\left(K^{2}\right) K^{\mu ; \nu} K_{\nu ; \mu} \\
& \left.+c_{4}\left(K^{2}\right) K^{\lambda} K_{; \lambda}^{\kappa} K^{\rho} K_{\kappa ; \rho}+\lambda\left(K^{2}+1\right)\right] \sqrt{-g} \mathrm{~d}^{4} x+S_{m}\left(\psi, g_{\mu \nu}\right),
\end{aligned}
$$

where $g=\operatorname{det}\left(g_{\mu \nu}\right)<0$ is the determinant of the metric tensor $g_{\mu \nu}, R$ is the Ricci scalar, $\psi$ denotes all the matter fields, $K^{2} \equiv K^{\lambda} K_{\lambda},-\delta_{\mu}^{0}$, which is Kronecker $\delta$, is the asymptotic value of $K_{\mu}$ and $c_{i}(i=1,2,3,4)$ are constants. The Lagrange multiplier $\lambda$ constrains the vector field $K^{2}$ to be -1 . Here, we respect the Einstein equivalence principle so that the

$\dagger$ This work was funded by the Natural Science Foundation of China. 
matter fields $\psi$ do not interact with the vector field, i.e. the action of matter, $S_{m}$, is the function of $\psi$ and $g_{\mu \nu}$ only.

In PN approximation, we consider an asymptotically flat spacetime, whose metric $g_{\mu \nu}$ has the form to second order as

$$
\begin{aligned}
& g_{00}=-1+\epsilon^{2} N+\epsilon^{4} L+\epsilon^{6} Q+\mathcal{O}\left(\epsilon^{8}\right) \\
& g_{0 i}=\epsilon^{3} L_{i}+\epsilon^{5} Q_{i}+\mathcal{O}\left(\epsilon^{7}\right), \\
& g_{i j}=\delta_{i j}+\epsilon^{2} H_{i j}+\epsilon^{4} Q_{i j}+\mathcal{O}\left(\epsilon^{6}\right),
\end{aligned}
$$

and an asymptotically unit time-like vector field, which is

$$
\begin{aligned}
K_{0} & =-1+\epsilon^{2} \stackrel{(2)}{K_{0}}+\epsilon^{4} \stackrel{(4)}{K_{0}}+\epsilon^{6} \stackrel{(6)}{K}_{0}+\mathcal{O}\left(\epsilon^{8}\right), \\
K_{i} & =\epsilon^{3} \stackrel{(3)}{K}_{i}+\epsilon^{5} \stackrel{(5)}{K}_{i}+\mathcal{O}\left(\epsilon^{7}\right),
\end{aligned}
$$

where $\epsilon \equiv 1 / c$. With the constraints of $K^{2}=-1$ and the harmonic gauge

$$
\left(\sqrt{-g} g^{\mu \nu}\right)_{, \nu}=0
$$

the $2 \mathrm{PN}$ metric coefficients for $N$ point masses have been worked out following the procedures proposed by Chandrasekhar (1965). The details can be found in Xie \& Huang (2007).

\section{2PN light propagation}

Light equations are (Will, 1993)

$$
g_{\mu \nu} \frac{\mathrm{d} x^{\mu}}{\mathrm{d} t} \frac{\mathrm{d} x^{\nu}}{\mathrm{d} t}=0
$$

and

$$
\frac{\mathrm{d}^{2} x^{i}}{\mathrm{~d} t^{2}}=\left(\frac{1}{c} \Gamma_{\mu \nu}^{0} \frac{\mathrm{d} x^{i}}{\mathrm{~d} t}-\Gamma_{\mu \nu}^{i}\right) \frac{\mathrm{d} x^{\mu}}{\mathrm{d} t} \frac{\mathrm{d} x^{\nu}}{\mathrm{d} t} .
$$

Assuming

$$
\vec{x}=\vec{x}_{0}+c \vec{n}\left(t-t_{0}\right)+\vec{x}_{1}+\vec{x}_{2},
$$

where $\vec{x}_{1}$ and $\vec{x}_{2}$ are $1 \mathrm{PN}$ and $2 \mathrm{PN}$ corrections, We have obtained the relevant light equation up to the $2 \mathrm{PN}$ approximation. If all the planets are ignored, the deflection of light to second order is

$$
\Delta \phi=\frac{4 \mathcal{G} M_{\odot}}{c^{2} d}+\left[\frac{\left(15+c_{14}\right) \pi}{4}-8\right] \frac{\mathcal{G}^{2} M_{\odot}^{2}}{c^{4} d^{2}},
$$

where $d$ represents the coordinate radius at the point of closest approach of the ray, $\mathcal{G}=2 G /\left(2-c_{14}\right)$ and $c_{14}=c_{1}+c_{4}$.

\section{References}

Chandrasekhar, S. 1965, ApJ, 142, 1488

Eling, C. \& Jacoboson, T. 2006, Class. Quant. Grav., 23, 5625; Class. Quant. Grav., 23, 5643

Garfinkle, D., Eling, C., \& Jacobson, T. 2007, Phys. Rev. D, 76, 024003

Jacoboson, T. \& Mattingly, D. 2001, Phys. Rev. D, 64, 024028; 2004, Phys. Rev. D, 70, 024003 Will, C. 1993, Theory and Experiment in Gravitational Physics, Cambridge Univ. Press.

Xie, Y., Ni, W.-T., Dong, P., \& Huang T.-Y. 2007, Adv. Sp. Res., accepted, doi:10.1016/j.asr.2007.09.022

Xie, Y. \& Huang T.-Y. 2007, in preparation. 